\title{
FOLIA 206
}

\section{Annales Universitatis Paedagogicae Cracoviensis Studia Mathematica XVI (2017)}

\section{Edward Tutaj \\ LikeN's - a point of view on natural numbers}

Communicated by Justyna Szpond

\begin{abstract}
We define and study some simple structures which we call likens and which are conceptually near to both sets of natural numbers, i.e. $\mathbb{N}$ with addition and $\mathbb{N}^{*}=\mathbb{N} \backslash\{0\}$ with multiplication. It appears that there are many different likens, which makes it possible to look on usual natural numbers from a more general point of view. In particular, we show that $\mathbb{N}$ and $\mathbb{N}^{*}$ are related to some functionals on the space of likens. A similar idea is known for a long time as the Beurling generalized numbers. Our approach may be considered as a little more natural and more general, since it admits the finitely generated likens.
\end{abstract}

\section{Introduction}

If we say the set of natural numbers we usually mean the set $\mathbb{N}=\{0,1,2, \ldots\}$ equipped with the operations of addition "+" and multiplication ".", and we mean the natural order in $\mathbb{N}$ which agree with both operations. The addition and multiplication are related by the distributive law: $k \cdot(m+n)=k \cdot m+k \cdot n$. If we reject the distributive law, we obtain two different structures: $(\mathbb{N},+)$ and $\left(\mathbb{N}^{*}, \cdot\right)$ which - as mathematical structures - are ordered semigroups. First of these semigroups $(\mathbb{N},+)$ is a sub-semigroup of the ordered semigroup $\mathbb{R}^{+}=[0,+\infty)$. This is not true for $\mathbb{N}^{*}=\{1,2,3, \ldots\}$ with multiplication. However the map

$$
\ln : \mathbb{N}^{*} \ni k \rightarrow \ln k \in \mathbb{R}^{+}
$$

is a monomorphism and is increasing. Hence the image $\ln \left(\mathbb{N}^{*}\right)$ is a sub-semigroup of $\mathbb{R}^{+}$. Here lies the essence of the idea of a liken: this is a sub-semigroup of $\mathbb{R}^{+}$ which is additionally ordered "like $\mathbb{N}$ ".

AMS (2010) Subject Classification: 11A41.

Keywords and phrases: Beurling numbers; distribution of prime numbers. 
The idea of the study of the structures, which are more general than the multiplicative semigroup of natural numbers, appeared for the first time eighty years ago in the paper of Beurling [1], with the aim to elucidate if, and how far, the Prime Number Theorem, describing the distribution of prime numbers in the set of natural numbers, depends on addition in $\mathbb{N}$. Since then several authors obtained many interesting theorems. An excellent review on these results is presented in the monograph [2]. To understand the definition of a liken, let us recall the definition of Beurling generalized numbers. Consider an increasing (not necessarily strictly) sequence $\mathbb{P}$ of real numbers

$$
1<p_{1} \leq p_{2} \leq p_{3} \ldots
$$

such that

$$
\lim _{n \rightarrow \infty} p_{n}=\infty .
$$

The numbers which can be written as

$$
n=p_{i_{1}}^{k_{1}} \cdot p_{i_{2}}^{k_{2}} \cdot p_{i_{m}}^{k_{m}}
$$

are called generalized integers and they form a multiplicative sub-semigroup $\mathbb{B}=$ $\mathbb{B}\left(p_{1}, p_{2}, \ldots\right)$ of the multiplicative semigroup $\mathbb{R}^{+}$. The elements of the sequence $\mathbb{P}=\left(p_{1}, p_{2}, \ldots\right)$ play the role of generalized prime numbers. The semigroup $\mathbb{B}$ is then generated by its subset $\mathbb{P}$ and one may then study the distribution of $\mathbb{P}$ in $\mathbb{B}$. Clearly, the family of sub-groups $\mathbb{B}$, which one may obtain in this way, is very rich, and hence one may observe different type of distribution of generalized primes in generalized integers. This is in fact the content of [2].

In the present paper, we would like to present a slightly different point of view on the Beurling numbers. First, it seems to be useful to consider the subgroups generated by a finite number of generators $\mathbb{B}\left(p_{1}, p_{2}, \ldots, p_{k}\right)$. Obviously, the problem of distribution of primes in such situation is not interesting, but the finitely generated sub-groups seem to be natural objects of this theory. Moreover, we think that because of the habits going from the linear algebra, it is most convenient to work with the additive sub-semigroups of the semigroup of positive reals. The aim we would like to reach, perhaps difficult to obtain, is to create a structure on the set of likens. We make in Part I of this paper some steps in this direction, where we define likens as countable semigroups which tend to infinity, and we make some general observations about likens. The main result of this part of the paper is Theorem 16 which says that two likens are isomorphic if and only if their generators are linearly dependent.

The second part of this paper is devoted to the study of the sequence of gaps in likens. Since a liken $\mathbb{L}=\left(x_{n}\right)_{0}^{\infty}$ is a strictly increasing sequence tending to infinity, one may consider the sequence of gaps of $\mathbb{L}$ defined as the sequence of differences $\delta_{k}=x_{k+1}-x_{k}$. We prove two theorems, Theorem 25 and Theorem 29 which describe some general properties of the sequence of gaps. In particular, Theorem 29 is a characterization of the additive set of natural numbers $\mathbb{N}$ among the family of all likens and this theorem may be confirming the usefulness of the space of likens. Indeed, it appears that only in $\mathbb{N}$ the sequence of gaps does not tend to 0 (each gap in $\mathbb{N}$ equals 1 ). The second theorem of this type, which will 
appear in the future work, says that, roughly speaking, the liken $\mathbb{N}^{*}$ is strongly related to the assumption that its sequence of gaps is strictly decreasing.

\section{Part I. General properties of likens}

\subsection{Definition of a liken}

In this paper, and in particular in this section, we will use the following notations:

$$
\begin{aligned}
\mathbb{R}^{+} & =[0, \infty), \\
\mathbb{Q}^{+} & =[0, \infty) \cap \mathbb{Q}, \\
\mathbb{R}^{\mathbb{N}} & =\left\{\vec{a}=\left(a_{i}\right)_{1}^{\infty}: a_{i} \in \mathbb{R}\right\}, \\
\left(\mathbb{R}^{+}\right)^{\mathbb{N}} & =\left\{\vec{a} \in \mathbb{R}^{\mathbb{N}}: a_{i} \geq 0\right\}, \\
\mathbb{R}_{0}^{\mathbb{N}} & =\left\{\vec{a} \in \mathbb{R}^{\mathbb{N}}: \exists j: i>j \Rightarrow a_{i}=0\right\}, \\
\mathbb{Q}^{\mathbb{N}} & =\left\{\vec{a}=\left(a_{i}\right)_{1}^{\infty}: a_{i} \in \mathbb{Q}\right\}, \\
\left(\mathbb{Q}^{+}\right)^{\mathbb{N}} & =\left\{\vec{a} \in \mathbb{Q}^{\mathbb{N}}: a_{i} \geq 0\right\}, \\
\mathbb{Q}_{0}^{\mathbb{N}} & =\left\{\vec{m} \in \mathbb{Q}^{\mathbb{N}}: \exists j: i>j \Rightarrow a_{i}=0\right\}, \\
\mathbb{N}_{0}^{\mathbb{N}} & =\left\{\vec{a} \in \mathbb{N}^{\mathbb{N}}: \exists j: i>j \Rightarrow a_{i}=0\right\} .
\end{aligned}
$$

We start by formulating the definition of a liken.

DEFINITION 1

A liken $\mathbb{L}$ is a sequence $\left(x_{n}\right)_{0}^{\infty}$ of real numbers such that:

a) for all $n \in \mathbb{N}$ we have $0=x_{0} \leq x_{n}<x_{n+1}$,

b) for all $m, n \in \mathbb{N}$ there is $k \in \mathbb{N}$ such that $x_{n}+x_{m}=x_{k}$.

It follows directly from Definition 1 that

\section{Proposition 2}

In the notations as above

i) $\mathbb{L}$ is a semigroup,

ii) $\lim _{n \rightarrow \infty} x_{n}=+\infty$.

Proof. The property i) follows directly from a) and b). Let us observe that a) implies inequality $0<x_{1}$, hence $\mathbb{L}$ is not trivial. We check by induction that for each $k \in \mathbb{N}$ the number $k \cdot x_{1} \in \mathbb{L}$. In other words, for each $k \in \mathbb{N}$ there exists $n_{k} \in \mathbb{N}$ such that $x_{n_{k}}=k \cdot x_{1}$. Hence $\left(x_{n}\right)_{0}^{\infty}$ is an increasing sequence which admits a subsequence $\left(x_{n_{k}}\right)_{1}^{\infty}$ tending to infinity. This ends the proof of Proposition 2.

Clearly, each liken is a countable sub-semigroup of $\mathbb{R}^{+}$, but the inverse is not true. The semigroup $\mathbb{Q}^{+}=[0, \infty) \cap \mathbb{Q}$ is a sub-semigroup of $\mathbb{R}^{+}$, but it is not a liken. Notice that the property ii) from Proposition 2 although formulated for 
a sequence $\left(x_{n}\right)_{0}^{\infty}$, is in fact a property of the set $\left\{x_{n}\right\}_{0}^{\infty}$ since the existence of the limit and its value are invariant under the permutations of $\mathbb{N}$. Hence equivalently one may say that a liken $\mathbb{L}$ is a countable sub-semigroup of $\mathbb{R}^{+}$which tends to $+\infty$. This is not the case of $\mathbb{Q}^{+}$. It will be convenient to use the notion of locally finite set. We recall that a subset $U$ of a topological space $X$ is locally finite in $X$ if for each point $x \in X$ there exists a neighbourhood $V$ of $x$ such that $V \cap U$ is a finite set. Using this notion, we may say shortly that the likens are locally finite sub-semigroups of $\mathbb{R}^{+}$. If $\mathbb{L}=\left(x_{n}\right)_{0}^{\infty}$ is a sub-semigroup of the subgroup of non-negative reals $\mathbb{R}^{+}$(clearly with the usual topology), then the sequence $y_{n}=\exp \left(x_{n}\right)$ tends to infinity and forms a multiplicative sub-semigroup of the multiplicative semigroup $[1, \infty)$. Conversely, if $\mathbb{G}=\left(x_{n}\right)_{1}^{\infty}$ is a strictly increasing sequence, which is a sub-semigroup of the multiplicative semigroup $[1, \infty)$, then the sequence $y_{n}=\ln \left(x_{n}\right)$ is a liken. As we will see later, there are no essential differences between likens and Beurling numbers. We will formulate all definitions and results for "additive" likens, but sometimes it will be more convenient to use the multiplicative notations.

In each liken we have at the moment two structures: an algebraic structure related to the addition and an ordinal structure related to the ordering in $\mathbb{R}$. Hence we must be precise, what we mean saying "isomorphism of likens". We formulate the formal definitions in order to avoid any misunderstanding.

DEFINITION 3

Let $(\mathcal{G},+)$ be a semigroup and let $\mathbb{L}$ be a liken. We will say that a map $\varphi: \mathcal{G} \rightarrow \mathbb{L}$ is

a) an algebraic homomorphism, when $\varphi(x+y)=\varphi(x)+\varphi(y)$,

b) an algebraic monomorphism, when it is an injective algebraic homomorphism,

c) an algebraic isomorphism, when it is a surjective algebraic monomorphism.

In particular, we know now, what it means that two likens $\mathbb{L}$ and $\mathbb{K}$ are algebraically isomorphic. It is also clear that each two likens are isomorphic as ordered spaces, since they are isomorphic to the ordered space $(\mathbb{N}, \leq)$. Let us mention that the map $\mathbb{N} \ni n \rightarrow x_{n} \in \mathbb{L}$ is not (in general) a homomorphism of likens and let us mention also that if $\varphi: \mathbb{K} \rightarrow \mathbb{L}$ is an ordinal isomorphism, then it is unique. Finally

DEFINITION 4

Two likens $\mathbb{L}$ and $\mathbb{K}$ are isomorphic if the (unique) ordinal isomorphism is also an algebraic homomorphism.

If $\mathbb{L}^{\prime}$ is a nontrivial subset of a liken $\mathbb{L}$, which is closed with respect to addition, then $\mathbb{L}^{\prime}$ is a liken too, and it is natural to say that $\mathbb{L}^{\prime}$ is a sub-liken of the liken $\mathbb{L}$.

In semigroups one can consider the so-called cancellation law, i.e. the following property of a semigroup $\mathbb{G}$ : for all $a \in \mathbb{G}, b \in \mathbb{G}, c \in \mathbb{G}$,

$$
a+c=b+c \Rightarrow b=c .
$$

It is well known, that for each semigroup $\mathbb{G}$ with the cancellation law there exists a group $\mathcal{G}$ (unique up to an isomorphism) such that, roughly speaking, 
$\mathcal{G}=\mathbb{G}-\mathbb{G}$. The reason for which we work with semigroups is that passing from $\mathbb{G}$ to $\mathcal{G}$, we loose the order which is "like in $\mathbb{N}$ ". The order will play a fundamental role in our considerations.

\subsection{Undecomposable elements}

An important consequence of the definition of a liken (more precisely, a consequence of the order type) is the existence of undecomposable elements. We will see that undecomposable elements in likens play an analogous role as prime numbers in $\mathbb{N}^{*}$.

\section{DEFINITION 5}

Let $\mathbb{L}$ be a liken and let $u \in \mathbb{L}$. We say that $u$ is undecomposable if

$$
u=v+w, v \in \mathbb{L} \ni w \Rightarrow v=0 \vee w=0 .
$$

Proposition 6

Each liken $\mathbb{L}=\left(x_{n}\right)_{0}^{\infty}$ has at least one undecomposable element.

Proof. We have observed earlier that $x_{1}>0$. We check that $u=x_{1}$ is undecomposable. Indeed, suppose that $x_{1}=v+w$, where $w$ and $v$ are from $\mathbb{L}$ and suppose that $v>0$ and $w>0$. This means in particular, that $0<v<x_{1}$ (and $0<w<x_{1}$ ). Thus $0<v<x_{1}=v+w$ which is impossible. This ends the proof.

\section{Proposition 7}

Let $\mathbb{L}$ be a liken, and let $P_{\mathbb{L}}$ be the set of indecomposable elements of $\mathbb{L}$. Then each element of $x \in \mathbb{L}$ can be written in the form

$$
x=m_{1} \cdot a_{1}+m_{2} \cdot a_{2}+\ldots+m_{k} \cdot a_{k},
$$

where $m_{1}, m_{2}, \ldots, m_{k} \in \mathbb{N}, a_{1}, a_{2}, \ldots, a_{k} \in P_{\mathbb{L}}$ and $k \in \mathbb{N}$.

Proof. Let $P_{\mathbb{L}}$ denote the set of all undecomposable elements of the liken $\mathbb{L}$. It follows from Proposition 6 that $P_{\mathbb{L}} \neq \emptyset$. The set $P_{\mathbb{L}}$ may have only one element, as is in the case of the liken $\mathbb{N}$ and $P_{\mathbb{L}}$ may have infinitely many elements, as in the case of the liken $\mathbb{N}^{*}$ (more exactly $\ln \left(\mathbb{N}^{*}\right)$, but we will write $N^{*}$ for $\ln \left(\mathbb{N}^{*}\right)$ ). Let $\mathbb{L}^{\prime}$ denote the set of all elements of $\mathbb{L}$ which can be represented in the form (2). Clearly, $\mathbb{L}^{\prime}$ is a sub-liken of $\mathbb{L}$. Suppose that $\mathbb{L} \backslash \mathbb{L}^{\prime} \neq \emptyset$. Let $y$ be the minimal element of $\mathbb{L} \backslash \mathbb{L}^{\prime}$. Since $P_{\mathbb{L}} \subset \mathbb{L}^{\prime}$ then $y \notin P_{\mathbb{L}}$. Hence $y$ can be written in the form $y=v+w$ where $v$ and $w$ are both non-trivial. This means that $v<y$ and $w<y$, and in consequence, by definition of $y, v$ and $w$ are in $\mathbb{L}^{\prime}$ which is impossible.

Proposition 7 says that the set $P_{\mathbb{L}}$ generates the liken $\mathbb{L}$. It is natural to ask now about the uniqueness of the representation (2). This question is a little more complicated, since we work with natural coefficients, and we will return to this question later. 


\subsection{Universal semigroup}

We will try now to answer the question: how rich the family of all likens is? In other words, we build something which one may call the space of likens.

Let $\mathbb{N}_{0}^{\mathbb{N}}$ denote, as in (1), the set of all sequences of natural numbers, with almost all terms vanishing, i.e.

$$
\mathbb{N}_{0}^{\mathbb{N}}:=\left\{\vec{n}=\left(n_{1}, n_{2}, \ldots\right):\left(n_{j} \in \mathbb{N}\right) \wedge\left(\exists i \in \mathbb{N}: k>i \Rightarrow n_{k}=0\right)\right\} .
$$

In the set $\mathbb{N}_{0}^{\mathbb{N}}$ we may consider the operations: +- addition and $\cdot-$ multiplication by natural numbers, defined as usually. With these operations $\mathbb{N}_{0}^{\mathbb{N}}$ is an algebraic structure which may be called semimodule or a cone over $\mathbb{N}$.

We set $e_{k}=(0,0, \ldots, 0,1,0, \ldots)$, i.e. $e_{k}$ is an element of $\mathbb{N}_{0}^{\mathbb{N}}$. So we have

$$
\vec{n}=\left(n_{1}, n_{2}, \ldots\right)=n_{1} \cdot e_{1}+n_{2} \cdot e_{2}+\ldots .
$$

Using the terminology from linear algebra, we may say that $\left(e_{k}\right)_{1}^{\infty}$ is a basis of the cone $\mathbb{N}_{0}^{\mathbb{N}}$. This means precisely that each element from $\mathbb{N}_{0}^{\mathbb{N}}$ can be, in a unique way, written as a linear combination of $\left(e_{k}\right)_{k \in \mathbb{N}}$ with the coefficient from $\mathbb{N}$. Clearly, $\mathbb{N}_{0}^{\mathbb{N}}$ is a semigroup. The structure of a cone over $\mathbb{N}$ is not specially important, but we will use this terminology because of the reasons which will become clear in the next section.

\subsection{Homomorphisms from $\mathbb{N}_{0}^{\mathbb{N}}$ to $\mathbb{R}^{+}$}

Clearly, $\mathbb{R}^{+}$is a cone over $\mathbb{N}$. A map $\varphi: \mathbb{N}_{0}^{\mathbb{N}} \rightarrow \mathbb{R}^{+}$will be called a homomorphism of semigroups, when

$$
\varphi\left(n_{1} \cdot e_{1}+n_{2} \cdot e_{2}+\ldots\right)=n_{1} \cdot \varphi\left(e_{1}\right)+n_{2} \cdot \varphi\left(e_{2}\right)+\ldots .
$$

It is evident that a homomorphism $\varphi: \mathbb{N}_{0}^{\mathbb{N}} \rightarrow \mathbb{R}^{+}$cannot be an epimorphism, (surjective homomorphism) since $\mathbb{N}_{0}^{\mathbb{N}}$ is countable (hence $\varphi\left(\mathbb{N}_{0}^{\mathbb{N}}\right)$ is countable too) and $\mathbb{R}^{+}$is uncountable. However there exist the monomorphisms (i.e. injective homomorphisms) $\varphi: \mathbb{N}_{0}^{\mathbb{N}} \rightarrow \mathbb{R}^{+}$. We will now give a more detailed description of this situation. The next proposition shows that the notion of a liken is practically equivalent to the notion the Beurling numbers.

\section{Proposition 8}

Each function $a: \mathbb{N} \rightarrow \mathbb{R}^{+}$can be extended in a unique way to a homomorphism $\tilde{a}: \mathbb{N}_{0}^{\mathbb{N}} \rightarrow \mathbb{R}^{+}$by linearity.

Proof. Let $a=\left(a_{i}\right)_{1}^{\infty}$. Setting $\tilde{a}\left(e_{i}\right)=a_{i}$, we obtain a necessary formula

$$
\tilde{a}\left(n_{1} \cdot e_{1}+n_{2} \cdot e_{2}+\ldots\right)=n_{1} \cdot a_{1}+n_{2} \cdot a_{2}+\ldots .
$$

It is not hard to check that $\tilde{a}$ is a homomorphism.

Let $\mathcal{H}\left(\mathbb{N}_{0}^{\mathbb{N}} ; \mathbb{R}^{+}\right.$) (we will write also simply $\mathcal{H}$ ) denote the set of all homomorphisms of the two considered cones. This set is also a cone with respect to the natural addition and the scalar multiplication. The same is true about the 
product $\left(\mathbb{R}^{+}\right)^{\mathbb{N}}$. It follows from Proposition 8 that these two structures (i.e. these two cones) are isomorphic and an isomorphism is for example given by

$$
\left(\mathbb{R}^{+}\right)^{\mathbb{N}} \ni a \rightarrow \tilde{a} \in \mathcal{H}\left(\mathbb{N}_{0}^{\mathbb{N}} ; \mathbb{R}^{+}\right) .
$$

On the other hand, if $\varphi \in \mathcal{H}$ then $a=\left(\varphi\left(e_{i}\right)\right)$ determines $\varphi$, i.e. $\varphi=\tilde{a}$. Hence we can identify the set of homomorphism $\mathcal{H}\left(\mathbb{N}_{0}^{\mathbb{N}} ; \mathbb{R}^{+}\right)$with the set $\left(\mathbb{R}^{+}\right)^{\mathbb{N}}$. In the last set we have a natural topology, i.e. the product topology which is metrisable. Usually one considers a metric given by

$$
d\left(\left(a_{k}\right) ;\left(b_{k}\right)\right)=\sum_{i=1}^{\infty} 2^{-i} \cdot \frac{\left|a_{i}-b_{i}\right|}{1+\left|a_{i}-b_{i}\right|} .
$$

Moreover, it is well known that $\left(\mathbb{R}^{+}\right)^{\mathbb{N}}$ with respect to this metric is a complete metric space (as a countable product of complete metric spaces). So, from the topological point of view, the structure of $\mathcal{H}\left(\mathbb{N}_{0}^{\mathbb{N}} ; \mathbb{R}^{+}\right)$is relatively simple.

The set of likens will appear to be a subset of $\mathcal{H}$. If $\varphi \in \mathcal{H}$ then $\varphi\left(\mathbb{N}_{0}^{\mathbb{N}}\right)$ is a countable sub-semigroup of $\mathbb{R}^{+}$, but is not a liken in general. For example suppose that $a=\left(a_{i}\right)_{1}^{\infty} \in \mathbb{R}^{+}$has a subsequence which has a finite positive limit. Then $\tilde{a}\left(\mathbb{N}_{0}^{\mathbb{N}}\right)$ cannot be a liken since $\left(a_{i}\right)_{1}^{\infty} \subset \tilde{a}\left(\mathbb{N}_{0}^{\mathbb{N}}\right)$ and in likens each element has only a finite number of preceding elements. To avoid some technical complications, we will consider separately the case of finitely generated likens.

\subsection{Likens with finite number of generators}

DeFinition 9

We will say that a liken $\mathbb{L}$ is finitely generated, when the set $P_{\mathbb{L}}$ is finite.

Since the set $P_{\mathbb{L}}$ is uniquely determined by $\mathbb{L}$, then so is the number card $\left(P_{\mathbb{L}}\right)$ which we call the dimension of $\mathbb{L}$. When $P_{\mathbb{L}}=\left\{a_{1}, a_{2}, \ldots, a_{k}\right\}$ then we write, if necessary, $\mathbb{L}=\mathbb{L}\left(a_{1}, a_{2}, \ldots, a_{k}\right)$.

As we observed in Proposition 7 each element of $x \in \mathbb{L}\left(a_{1}, a_{2}, \ldots, a_{k}\right)$ can be written in the form

$$
x=m_{1} \cdot a_{1}+m_{2} \cdot a_{2}+\ldots+m_{k} \cdot a_{k},
$$

but unfortunately in likens the representation (4) in general is not unique, as we have in the case of bases of vector spaces.

EXAMPLE 10

Let $a_{1}=1, a_{2}=\sqrt{2}$ and let $a_{3}=1+\frac{\sqrt{2}}{2}$. It is easy to check that $a_{3} \notin \mathbb{L}\left(a_{1}, a_{2}\right)$ but $2 \cdot a_{3}=2 \cdot a_{1}+a_{2}$. In other words, in the liken $\mathbb{L}\left(a_{1}, a_{2}, a_{3}\right)$ the uniqueness does not hold.

Hence if we wish to have a liken, in which the representation (4) is unique, we must do an additional assumption. Let us denote the vector space $\mathbb{R}$ over the field $\mathbb{Q}$ by $(\mathbb{R}, \mathbb{Q})$. We have the obvious fact 


\section{Proposition 11}

If the real numbers $a_{1}, a_{2}, \ldots, a_{k}$ are linearly independent in the vector space $(\mathbb{R}, \mathbb{Q})$, then the representation (4) is unique.

The likens, which have the property described in Proposition 11, will be called in the sequel likens with uniqueness.

A little more complicated is the following observation

Proposition 12

For any finite sequence of positive real numbers $u_{1}, u_{2}, \ldots, u_{k}$, the set $\mathbb{L}$ of all real numbers $x$, which can be written in the form

$$
x=m_{1} \cdot u_{1}+m_{2} \cdot u_{2}+\ldots+m_{k} \cdot u_{k},
$$

where $m_{1}, m_{2}, \ldots, m_{k} \in \mathbb{N}$, is a liken.

Proof. Clearly, $\mathbb{L}$ is a semigroup, then it remains to show that $\mathbb{L}$ tends to $+\infty$. Equivalently, we must show that for each $A>0$ the set $\{x \in \mathbb{L}: x \leq A\}$ is finite. Let us fix a number $A$ and let $\alpha=\min \left\{u_{1}, u_{2}, \ldots, u_{k}\right\}$. Thus $\alpha>0$. Let $n=E\left(\frac{A}{\alpha}\right)+1$, where $E(z)$ is the integral part of the real number $z$. It is easy to check that the cardinality of the set $\{x \in \mathbb{L}: x \leq A\}$ is less than $n^{k}$.

We will describe a topological character of the space of finitely generated likens with uniqueness. Let $\mathcal{L}^{k}$ denote the set of all likens with uniqueness which have exactly $k$ undecomposable elements. It follows from Propositions 7, 11, and 12 that $\mathcal{L}^{k}$ can be identified with the set $\mathcal{M}^{k}$ of all increasing sequences of positive reals $\vec{a}=\left(a_{1}, a_{2}, \ldots, a_{k}\right)$ which are linearly independent in the vector space $(\mathbb{R}, \mathbb{Q})$. Let $M^{k}$ denote the cone of all non-negative and non-decreasing sequences from $\left(\mathbb{R}^{+}\right)^{k}$. It is easy to observe that $M^{k}$ is a closed sub-cone of $\left(\mathbb{R}^{+}\right)^{k}$ and $M^{k}$ has non-empty interior. We have the following fact

Proposition 13

The set $\mathcal{M}^{k}$ is a $G_{\delta}$ dense subset of $M^{k}$.

Proof. Let $\vec{m}=\left(m_{1}, m_{2}, \ldots, m_{k}\right)$ be a point from $\mathbb{Z}^{k}$ and let

$$
M_{\vec{m}}=\left\{\vec{a} \in\left(\mathbb{R}^{+}\right)^{k}: m_{1} a_{1}+m_{2} a_{2}+\ldots+m_{k} a_{k}=0\right\} .
$$

It is clear that $M_{\vec{m}}$ is a closed subset of $\left(\mathbb{R}^{+}\right)^{k}$ and has empty interior. It is also easy to check that

$$
\mathcal{M}_{k}=M_{k} \backslash \bigcup_{\vec{m} \in \mathbb{Z}^{k}} M_{\vec{m}}
$$

This ends the proof, since $\mathbb{Z}^{k}$ is countable.

\subsection{Likens with infinite number of generators}

Now we will give an analogous description of the set of infinitely generated likens. Let $\mathbb{L}$ be a liken and let the set $P_{\mathbb{L}}=\left\{a_{1}, a_{2}, \ldots\right\}$ be infinite. We assume that $P_{\mathbb{L}}$ is linearly independent in the vector space $(\mathbb{R}, \mathbb{Q})$. This assumption is sufficient to have the uniqueness of the representation (2). We have observed in Proposition 2 that in the case when $P_{\mathbb{L}}$ is infinite we must have $\lim _{k \rightarrow \infty} a_{k}=+\infty$. The converse is also true. Namely, the following proposition holds 


\section{Proposition 14}

Let $\vec{a}=\left(a_{i}\right)_{1}^{\infty}$ be a sequence from $\left(\mathbb{R}^{+}\right)^{\mathbb{N}}$ which is linearly independent in $(\mathbb{R}, \mathbb{Q})$ and tends to infinity. Then $\tilde{a}\left(\mathbb{N}_{0}^{\mathbb{N}}\right)$ is a liken.

Proof. Since the image $\tilde{a}\left(\mathbb{N}_{0}^{\mathbb{N}}\right)$ does not change after permuting the set $P_{\mathbb{L}}$ and since $\vec{a}=\left(a_{1}, a_{2}, \ldots\right)$ tends to infinity, we may assume that $\left(a_{k}\right)_{1}^{\infty}$ is increasing and that $a_{1}>0$. Let $A>0$. We must show that only a finite number of members of the set $\tilde{a}\left(\mathbb{N}_{0}^{\mathbb{N}}\right)$ is less than $A$. Let $j \in \mathbb{N}$ be a natural number such that $k>j \Rightarrow a_{k}>A$. Let $\vec{m}=\left(m_{1}, m_{2}, \ldots\right)$ be a point from $\mathbb{N}_{0}^{\mathbb{N}}$ such that the following is true:

i) there is $k_{0}>j$ such that $m_{k_{0}} \geq 1$,

or

ii) there is $k_{0} \leq j$ such that $m_{k_{0}}>\frac{A}{a_{1}}$.

If i) then

$$
\tilde{a}(\vec{m})=m_{1} a_{1}+m_{2} a_{2}+\ldots+m_{j} a_{j}+\ldots+m_{k_{0}} a_{k_{0}} \ldots>m_{k_{0}} a_{k_{0}}>A .
$$

If ii) then

$$
\tilde{a}(\vec{m})=m_{1} a_{1}+\ldots+m_{k_{0}} a_{k_{0}}+\ldots+m_{j} a_{j}+\ldots>m_{k_{0}} a_{k_{0}}>a_{1} \cdot \frac{A}{a_{1}}>A .
$$

This implies that the only elements of type $\tilde{a}(\vec{m})$ which can be less than $A$ are those with $m_{k}=0$ for $k>j$ (since $m_{k}$ is a natural number, $m_{k}<1$ implies $\left.m_{k}=0\right)$ and $m_{k}$ are equi-bounded for $k \leq j$. But the number of such $\vec{m}$ is finite.

Proposition 14 makes it possible to prove for likens with infinitely many generators and with uniqueness, a theorem similar to Proposition 13 More exactly, we have

\section{Proposition 15}

Let $\mathcal{L}^{\infty}$ denote the set of all likens with infinite number of generators and with uniqueness. Then $\mathcal{L}^{\infty}$ is a $G_{\delta}$ dense subset in an infinite dimensional complete metric space.

We omit the proof of Proposition 15 since it is similar to the proof of Proposition 13

\subsection{A theorem on isomorphism of likens}

Suppose that we have two sequences $\vec{a}=\left(a_{k}\right)_{1}^{\infty}$ and $\vec{b}=\left(b_{k}\right)_{1}^{\infty}$ which generate two likens with uniqueness denoted by $\mathbb{L}_{a}$ and $\mathbb{L}_{b}$, respectively. We will prove the following

THEOREM 16

In the notations as above the likens $\mathbb{L}_{a}$ and $\mathbb{L}_{b}$ are isomorphic, if and only if there exists a positive number $\lambda$ such that $\vec{a}=\lambda \cdot \vec{b}$. 
Before starting the proof of this theorem, we must do some preparatory observations.

Clearly, when $\vec{a}=\lambda \cdot \vec{b}$, then $\mathbb{L}_{a}$ is isomorphic to $\mathbb{L}_{b}$. So we must prove that if $\mathbb{L}_{a}$ is isomorphic to $\mathbb{L}_{b}$, then $\vec{a}=\lambda \cdot \vec{b}$ for some $\lambda>0$. Suppose then that $\varphi: \mathbb{L}_{a} \rightarrow \mathbb{L}_{b}$ is an isomorphism (algebraic and ordinal) of likens. The same is true for the map $\varphi^{-1}: \mathbb{L}_{b} \rightarrow \mathbb{L}_{a}$. First we observe that for each $k$ the element $\varphi\left(a_{k}\right) \in \mathbb{L}_{b}$ is undecomposable in $\mathbb{L}_{b}$. Indeed, if $\varphi\left(a_{k}\right)=t+s$ implies $a_{k}=\varphi^{-1}(t)+\varphi^{-1}(s)$ and thus if $t$ and $s$ are both non-trivial, then so are $\varphi^{-1}(t)$ and $\varphi^{-1}(s)$.

Next, by induction we check that for each $k$ the equality $\varphi\left(a_{k}\right)=b_{k}$ holds. In consequence for each $\vec{m}=\left(m_{1}, m_{2}, \ldots\right) \in \mathbb{N}_{0}^{\mathbb{N}}$ we have

$$
\varphi\left(m_{1} \cdot a_{1}+m_{2} \cdot a_{2}+\ldots\right)=m_{1} \cdot b_{1}+m_{2} \cdot b_{2}+\ldots
$$

or equivalently for each $\vec{m} \in \mathbb{N}_{0}^{\mathbb{N}}$,

$$
\varphi(\tilde{a}(\vec{m}))=\tilde{b}(\vec{m})
$$

and finally we may say that for each $\vec{m} \in \mathbb{N}_{0}^{\mathbb{N}} \ni \vec{n}$ we have

$$
\tilde{a}(\vec{m}) \leq \tilde{a}(\vec{n}) \Leftrightarrow \tilde{b}(\vec{m}) \leq \tilde{b}(\vec{n})
$$

In other words, we have just proved that the condition $\mathbb{L}_{a}$ isomorphic to $\mathbb{L}_{b}$ implies the condition (5).

Let us observe that each element $\vec{a} \in \mathbb{R}^{\mathbb{N}}=\left\{\vec{a}=\left(a_{i}\right)_{1}^{\infty}: a_{i} \in \mathbb{R}\right\}$ defines a linear functional on the vector space $\mathbb{R}_{0}^{\mathbb{N}}=\left\{\vec{a}: \exists j: i>j \Rightarrow a_{i}=0\right\}$ given by the following formula

$$
a(x):=\langle\vec{a}, \vec{x}\rangle=\sum_{1}^{\infty} a_{i} \cdot x_{i}
$$

The series in (6) is convergent since each vector $x \in \mathbb{R}_{0}^{\mathbb{N}}$ has only a finite number of non-zero coordinates.

DeFinition 17

Given a space (set) $\mathcal{F} \subset \mathbb{R}^{\mathbb{N}}$ and two linear functionals $\vec{a}$ and $\vec{b}$, we will say that these functionals agree with respect to the order on the space $\mathcal{F}$, when for each $x, y \in \mathcal{F}$ the following equivalence holds

$$
a(x) \leq a(y) \Leftrightarrow b(x) \leq b(y) .
$$

We will prove the following

THEOREM 18
If two functionals $\vec{a}$ and $\vec{b}$ agree with respect to the order on $\mathbb{N}_{0}^{\mathbb{N}}$ then they agree with respect to the order on $\mathbb{R}^{\mathbb{N}}$. 
Proof. Let us assume that $\vec{a}$ and $\vec{b}$ agree with respect to the order on $\mathbb{N}_{0}^{\mathbb{N}}$.

i) We will prove that $\vec{a}$ and $\vec{b}$ agree with respect to the order on $\left(\mathbb{Q}^{+}\right)_{0}^{\mathbb{N}}$. Let us assume that $x$ and $y$ are two vectors from $\left(\mathbb{Q}^{+}\right)_{0}^{\mathbb{N}}$. Then there exists a natural number $\beta>0$ such that the vectors $x^{\prime}=\beta x$ and $y^{\prime}=\beta y$ are in $\mathbb{N}_{0}^{\mathbb{N}}$. Hence

$$
\begin{aligned}
a(x) \leq a(y) & \Leftrightarrow \beta a(x) \leq \beta a(y) \Leftrightarrow a(\beta x) \leq a(\beta y) \Leftrightarrow a\left(x^{\prime}\right) \leq a\left(y^{\prime}\right) \\
& \Leftrightarrow a\left(x^{\prime}\right) \leq a\left(y^{\prime}\right) \Leftrightarrow b\left(x^{\prime}\right) \leq b\left(y^{\prime}\right) \Leftrightarrow \beta b(x) \leq \beta b(y) \\
& \Leftrightarrow b(x)<b(y) .
\end{aligned}
$$

Thus i) is proved.

ii) Now we will prove that $\vec{a}$ and $\vec{b}$ agree on $\left(\mathbb{R}^{+}\right)_{0}^{\mathbb{N}}$. Indeed, suppose that $x$ and $y$ are two vectors from $\left(\mathbb{R}^{+}\right)_{0}^{\mathbb{N}}$. We may assume, without loss of generality, that $x$ and $y$ have both a finite support bounded by a number $k \in \mathbb{N}$. Then there exist two sequences of vectors $x_{n}$ and $y_{n}$ from $\left(\mathbb{Q}^{+}\right)_{0}^{\mathbb{N}}$ such that $x_{n}$ tends to $x$ and $y_{n}$ tends to $y$ and all elements of these two sequences have the supports also bounded by $k$. Hence we have a sequence of equivalences

$$
a\left(x_{n}\right) \leq a\left(y_{n}\right) \Leftrightarrow b\left(x_{n}\right) \leq b\left(y_{n}\right) .
$$

Since the supports of all considered vectors are commonly bounded, we may pass to the limit and we obtain $a(x) \leq a(y) \Leftrightarrow b(x) \leq b(y)$.

iii) Suppose now that $z \in \mathbb{R}_{0}^{\mathbb{N}}$ is an arbitrary vector. We have the following equivalence

$$
a(z) \leq 0 \Leftrightarrow b(z) \leq 0 .
$$

Indeed $z=z^{+}-z^{-}$, where $z^{+}$and $z^{-}$denote the positive and negative parts of the vector $z$, respectively. Thus we have

$$
\begin{aligned}
a(z) \leq 0 & \Leftrightarrow a\left(z^{+}-z^{-}\right) \leq 0 \Leftrightarrow a\left(z^{+}\right) \leq a\left(z^{-}\right) \Leftrightarrow b\left(z^{+}\right) \leq b\left(z^{-}\right) \\
& \Leftrightarrow b\left(z^{+}-z^{-}\right) \leq 0 \leq b(z) \leq 0 .
\end{aligned}
$$

This ends the proof of iii) since for each two non-negative vectors $x$ and $y$ we have

$$
a(x)<a(y) \Leftrightarrow a(x-y)<0 \Leftrightarrow b(x-y)<0 \Leftrightarrow b(x)<b(y) .
$$

It follows from the above considerations that

THEOREM 19

If two functionals $\vec{a}$ and $\vec{b}$ agree with respect to the order on $\mathbb{N}_{0}^{\mathbb{N}}$ then they are linearly dependent.

Proof. Applying Theorem 18, it follows from our assumptions that our functionals agree with respect to the order on $\mathbb{R}_{0}^{\mathbb{N}}$. But this means that $\vec{a}$ and $\vec{b}$ have equal 
kernels. Indeed, suppose that for an $x \in \mathbb{R}_{0}^{\mathbb{N}}$ we have $a(x)=0$. Since by Theorem 18.

$$
a(x)=a(0) \Leftrightarrow b(x)=b(0)
$$

then $b(x)=0$. It is known that two functionals are linearly dependent if and only if their kernels are equal.

Now we are ready to prove Theorem 16 .

Proof. We have observed above in formula (5) that if $\mathbb{L}_{a}$ and $\mathbb{L}_{b}$ are isomorphic, then the functionals $\vec{a}$ and $\vec{b}$ agree with respect to the order and then by Theorem 19. $\vec{a}$ and $\vec{b}$ are linearly dependent.

We will end this section by the following remark.

REMARK 20

As we have observed above, given a set of generators (finite, or infinite) - say $\vec{a}=\left(a_{k}\right)_{1}^{\infty}$ - the liken $\mathbb{L}_{a}$ does not depend on the sequence $\left(a_{k}\right)_{1}^{\infty}$ but depends only on the set of its elements. The unique property we need is to be locally finite. Clearly, each finite set is locally finite, and for infinite sets $U$ (subset of $U \subset \mathbb{R}^{+}$) we know that $U$ is locally finite if and only if $U$ tends to $+\infty$.

\subsection{Different counting functions in likens}

Suppose that $\vec{a}=\left(a_{i}\right)_{i=1}^{\infty}$ (or $\vec{a}=\left(a_{i}\right)_{i=1}^{k}$ ) defines a monomorphism. This means that $\tilde{a}\left(\mathbb{N}_{0}^{\mathbb{N}}\right)$ is a strictly increasing sequence $\mathbb{L}_{a}=\left(x_{n}\right)_{n=0}^{\infty}$. As we have observed above $\left(a_{i}\right)_{1}^{\infty}\left(\right.$ or $\left.\left(a_{i}\right)_{1}^{k}\right)$ is a subsequence of the sequence $\left(x_{n}\right)_{n=0}^{\infty}$, and an element $x_{n} \in \mathbb{L}_{a}$ is undecomposable if and only if $x_{n}=\tilde{a}\left(e_{i}\right)$ for some $i \in \mathbb{N}$ $(i \leq k)$. In other words, only those $x_{n}$ are undecomposable (we will also say prime) which are equal to some $a_{i}$.

The fact that $\mathbb{L}_{a}$ behaves "like $\mathbb{N}$ " makes possible to define and study different counting functions, similar to the well known prime counting function in $\mathbb{N}^{*}$.

DEFINITION 21

For $\vec{a}$ and for $\mathbb{L}_{a}$ as above and for a real number $x \in \mathbb{R}$ we set

$$
\pi_{\mathbb{L}}(x)=\operatorname{card}\left\{n \in \mathbb{N}: x_{n} \leq x\right\} .
$$

Hence the function $\pi_{\mathbb{L}}(x)$ counts the number of those elements of the sequence $x_{n}$ which are less than $x$. Unfortunately, it is very difficult to write down precisely the formula for the function $\pi_{\mathbb{L}}$ for a given liken $\mathbb{L}$. However, in two important situations we can do it. If $\mathbb{L}=\mathbb{N}$, i.e. $\mathbb{L}$ has one generator equal 1 , then $\pi_{\mathbb{L}}(x)=$ $E(x)$, where $E(x)$ is integral part of $x$. If $\mathbb{L}=\mathbb{N}^{*}$ then, as we have observed earlier, $x_{n}=\ln (n)$. This implies that

$$
\pi_{\mathbb{L}}(x)=E\left(e^{x}\right) .
$$

The next functions we are going to define, are directly related with the undecomposable elements. Let $\mathbb{L}=\left(x_{n}\right)_{0}^{\infty}$ be a liken, and let $\left(a_{k}\right)_{1}^{\infty}$ be a sequence (increasing) of undecomposable elements of $\mathbb{L}$. For $x \in \mathbb{R}$ we set

$$
\pi_{a}(x)=\max \left\{k: a_{k} \leq x\right\}
$$


and

$$
\pi_{a}^{*}(m)=\pi_{a}\left(x_{m}\right)
$$

This last function counts the number of generators, as a function of the index $n$, of the term $x_{n}$. It may be considered as a generalization of the prime counting function in the case of the classical liken $\mathbb{N}^{*}$ which is, as we know, asymptotically equal to $\frac{n}{\ln (n)}$.

The next theorem is rather rough, but gives some qualitative information about the function $\pi_{\mathbb{L}}$ in the case, when $\mathbb{L}$ is finitely generated.

\section{TheOREM 22}

Suppose that $0<a_{1}<a_{2}<\ldots<a_{k}$ is a sequence of real numbers which are linearly independent in the vector space $(\mathbb{R}, \mathbb{Q})$. Let $\mathbb{L}$ be a liken generated by the sequence $\left(a_{i}\right)_{1}^{k}$, i.e. $\mathbb{L}=\mathbb{L}\left(a_{1}, a_{2}, \ldots, a_{k}\right)$. Then there exist two polynomials, $w_{\mathbb{L}}$ and $W_{\mathbb{L}}($ depending on $\mathbb{L})$ of degree $k$ such that

$$
w_{\mathbb{L}}(x) \leq \pi_{\mathbb{L}}(x) \leq W_{\mathbb{L}}(x) .
$$

Proof. The isomorphism $\tilde{a}$ given by (3) defining liken $\mathbb{L}\left(a_{1}, a_{2}, \ldots, a_{k}\right)$ is now defined on $\mathbb{N}^{k}=\mathbb{N} \times \mathbb{N} \times \ldots \times \mathbb{N}$ and has the form

$\tilde{a}: \mathbb{N} \times \mathbb{N} \times \ldots \times \mathbb{N} \ni\left(m_{1}, m_{2}, \ldots, m_{k}\right) \rightarrow m_{1} \cdot a_{1}+m_{2} \cdot a_{2}+\ldots+m_{k} \cdot a_{k}=\tilde{a}(\vec{m}) \in \mathbb{R}$

Since $\mathbb{N}^{k} \subset \mathbb{R}^{k}$ then we may consider the linear functional on $\mathbb{R}^{k}$ given by $\tilde{a}(\vec{x})=$ $\sum_{i=1}^{k} a_{i} \cdot x_{i}$. The points $\vec{m} \in \mathbb{N} \times \mathbb{N} \times \ldots \times \mathbb{N}$ will be called lattice points. When a real number $x \in[0, \infty)$ moves, then the hyperplane

$$
H_{x}=\left\{\vec{x} \in \mathbb{R}^{k}: \tilde{a}(\vec{x})=x\right\}
$$

moves in $\mathbb{R}^{k}$ and in each position for $x>0$ cuts the cone $\left(\mathbb{R}^{k}\right)^{+}$and forms a pyramid, or - more precisely - a simplex

$$
\left.\mathbb{S}_{x}=\left\{\vec{x} \in \mathbb{R}^{k}\right)^{+}: 0 \leq \tilde{a}(\vec{x}) \leq x\right\}
$$

Now we see that the number $\pi_{\mathbb{L}}(x)$ equals to the number of lattice points in $\mathbb{S}_{x}$. To evaluate from the above the number of lattice points in $\mathbb{S}_{x}$, we consider a rectangular parallelepiped formed by the edges of $\mathbb{S}_{x}$ "starting" from the origin. If we denote this rectangular parallelepiped by $\mathbb{P}_{x}$, then since $\mathbb{S}_{x} \subset \mathbb{P}_{x}$, we conclude that $\pi_{\mathbb{L}}(x)$ is less than the number of lattice points in $\mathbb{P}_{x}$. The length of the edge of $\mathbb{S}_{x}$ lying on the axis generated by $e_{i}=(0,0, \ldots, 1,0, \ldots, 0)$ equals $\frac{x}{a_{i}}$, hence the number of lattice points in $\mathbb{P}_{x}$ is less than the volume of $\mathbb{P}_{x}$. This implies that as $W_{\mathbb{L}}$ we can take the polynomial

$$
W_{\mathbb{L}}(x)=\frac{x^{k}}{a_{1} \cdot a_{2} \cdot \ldots \cdot a_{k}} .
$$

To evaluate $\pi_{\mathbb{L}}(x)$ from below, let us observe that there exists a number $d>0$ (depending on $a_{1}, a_{2}, \ldots, a_{k}$ ) such that $[0, d]^{k} \subset \mathbb{S}_{1}$. In consequence, the simplex $\mathbb{S}_{x}$ contains the product $[0, d \cdot x]^{k}$ and thus $\pi_{\mathbb{L}}(x)$ is bounded from below by the 
number of lattice points in $[0, d \cdot x]^{k}$. This implies that as $w_{\mathbb{L}}$ we can take the polynomial

$$
w_{\mathbb{L}}(x)=(d \cdot x-1)^{k} .
$$

The polynomial $W_{\mathbb{L}}$ bounds the counting function $\pi_{\mathbb{L}}$ from above, but is far from to be the best upper bound, since the rectangular parallelepiped $\mathbb{P}_{x}$ is considerably bigger than the prism $\mathbb{S}_{x}$. The right order of magnitude of $\pi_{\mathbb{L}}(x)$ at infinity is near rather to the Lebesgue measure $m_{k}\left(\mathbb{S}_{x}\right)$ which equals

$$
m_{k}\left(\mathbb{S}_{x}\right)=\frac{1}{k !} \cdot \frac{x^{k}}{a_{1} \cdot a_{2} \cdot \ldots \cdot a_{k}} .
$$

The problem of finding the exact number of lattice points in $\mathbb{S}_{x}$ is a complicated problem from the discrete geometry (counting lattice points) and the so-called Ehrhart polynomials [3]. However, even this rough information, which is given by Theorem 22 allows to the following

COROLlary 23

The set of prime numbers in $\mathbb{N}^{*}$ is infinite.

Proof. Indeed, as we have observed above, the liken $\mathbb{N}^{*}$ has an exponential counting function which cannot be controlled from above by any polynomial. Hence $\mathbb{N}^{*}$ cannot be finitely generated.

\section{Part II. Gaps in likens}

The word gap is frequently used to name the difference between two successive elements of a given sequence. In this section we will prove two theorems about the gaps in likens.

\subsection{First theorem}

If $\mathbb{L}=\left(x_{n}\right)_{0}^{\infty}$ is a liken, then $\left(x_{n}\right)_{0}^{\infty}$ is strictly increasing and then injective. It seems to be interesting to observe that the sequence of gaps between the elements of $\mathbb{L}$ in general must not be injective. In particular, this is the case of finitely generated likens.

\section{DEFINITION 24}

Let $L=L\left(a_{1}, a_{2}, \ldots, a_{d}\right)=\left(x_{n}\right)_{0}^{\infty}$ be a liken with $d$ generators $a_{1}, a_{2}, \ldots, a_{d}$ which are independent in the vector space $(\mathbb{R}, \mathbb{Q})$. The sequence of differences $\delta:=\left(\delta_{k}\right)_{k=0}^{\infty}$,

$$
\delta_{k}=x_{k+1}-x_{k}
$$

will be called the sequence of gaps of the liken $\mathbb{L}$.

Clearly, by definition of a liken, $\delta_{k}>0$, since $L$ is strictly increasing. 
Let us fix $x>0$ and let $M(x)=\left\{x_{n} \in L: x_{n} \leq x\right\}$. Hence

$$
M(x)=\left\{x_{0}, x_{1}, x_{2}, \ldots, x_{k(x)}\right\},
$$

where $x_{0}<x_{1}<x_{2}<\ldots<x_{k(x)}$ and $k(x)=\max \left\{n: x_{n} \leq x\right\}$. We see that $k(x)=\pi_{L}(x)$ (7).

We set

$$
\pi_{\delta}(x)=\operatorname{card}(\delta(\{0,1,2, \ldots, k(x)\})) .
$$

In the notations as above we have

\section{THEOREM 25}

There exists a polynomial $W_{d}$ such that $\operatorname{deg}\left(W_{d}\right)<d$ and such that $\pi_{\delta}(x) \leq W_{d}(x)$.

Proof. It is clear that Theorem 25 is true in the case, when $d=1$ (one can take $W_{1} \equiv 1$ ), hence in the sequel we may assume that $d \geq 2$.

It follows from our assumptions on $\mathbb{L}$ that each element $x_{k}$ of the liken $\mathbb{L}$ can be uniquely represented in the form (2),

$$
x_{k}=m_{k}^{1} \cdot a_{1}+m_{k}^{2} \cdot a_{2}+\ldots+m_{k}^{d} \cdot a_{d},
$$

where $m_{k}^{1}, m_{k}^{2}, \ldots, m_{k}^{d} \in \mathbb{N}$. Hence we have

$$
\delta_{k}=\sum_{i=1}^{d} m_{k+1}^{i} \cdot a_{i}-\sum_{i=1}^{d} m_{k}^{i} \cdot a_{i}=\sum_{i=1}^{d}\left(m_{k+1}^{i}-m_{k}^{i}\right) \cdot a_{i} .
$$

Putting $\epsilon_{k}^{i}=m_{k+1}^{i}-m_{k}^{i}$, we can write

$$
\delta_{k}=\sum_{i=1}^{d} \epsilon_{k}^{i} \cdot a_{i},
$$

where all $\epsilon_{k}^{i}$ are integral numbers.

Some of the numbers $\epsilon_{k}^{i}$ are positive, some may be negative. Since the numbers $\delta_{k}$ are all positive then for each $k$ at least one of the numbers $\epsilon_{k}^{j}$ is positive. We set $I_{d}=I=\{1,2, \ldots, d\}$ and we denote

$$
A_{k}=\left\{j \in I_{d}: \epsilon_{k}^{j}>0\right\}
$$

and

$$
A_{k}^{\prime}=I_{d} \backslash A_{k} .
$$

Setting $n_{k}^{j}=\epsilon_{k}^{j}$ for $j \in A_{k}$ and $n_{k}^{j}=-\epsilon_{k}^{j}$ for $j \in I_{d} \backslash A_{k}$ we can write

$$
\delta_{k}=\sum_{j \in A_{k}} n_{k}^{j} \cdot a_{j}-\sum_{j \in A_{k}^{\prime}} n_{k}^{j} \cdot a_{j}
$$

Let us observe now that the set $A_{k}^{\prime}$ cannot be empty. Indeed, suppose that for each $j$ we have $\epsilon_{k}^{j}>0$, or equivalently, that $m_{k+1}^{i}-m_{k}^{i}>0$. Hence, in particular, $(d \geq 2) \epsilon_{k}^{1}>0$ and $\epsilon_{k}^{2}>0$. Thus $m_{k+1}^{1} \geq m_{k}^{1}+1$ and $m_{k+1}^{2} \geq m_{k}^{2}+1$. This implies 


$$
\begin{aligned}
x_{k+1} & \geq m_{k+1}^{1} \cdot a_{1}+m_{k+1}^{2} \cdot a_{2}+\sum_{j>2} m_{k+1}^{j} \cdot a_{j} \\
& >m_{k}^{1} \cdot a_{1}+m_{k+1}^{2} \cdot a_{2}+\sum_{j>2} m_{k+1}^{j} \cdot a_{j} \\
& >m_{k}^{1} \cdot a_{1}+m_{k}^{2} \cdot a_{2}+\sum_{j>2} m_{k+1}^{j} \cdot a_{j} \\
& \geq m_{k}^{1} \cdot a_{1}+m_{k}^{2} \cdot a_{2}+\sum_{j>2} m_{k}^{j} \cdot a_{j} \\
& =x_{k} .
\end{aligned}
$$

Setting

$$
z=m_{k}^{1} \cdot a_{1}+m_{k+1}^{2} \cdot a_{2}+\sum_{j>2} m_{k+1}^{j} \cdot a_{j}
$$

we see that $x_{k+1}>z>x_{k}$. Hence $x_{k}$ and $x_{k+1}$ cannot be two successive elements of the liken $L$. In other words, this means that the set $A_{k}$ is never empty and is never all $I_{d}$, or equivalently $0<\operatorname{card} A_{k}<d$.

Let

$$
\mathcal{P}_{d}=\left\{A \subset I_{d}: A \neq \emptyset, A \neq I_{d}\right\} .
$$

The family $\mathcal{P}_{d}$ has $2^{d}-2$ elements. It follows from the above that for each $k \in \mathbb{N}$ there exists a set $A \in \mathcal{P}_{d}$ such that we can rewrite (8),

$$
\delta_{k}=\sum_{j \in A} n_{k}^{j} \cdot a_{j}-\sum_{j \in A^{\prime}} n_{k}^{j} \cdot a_{j} .
$$

Let us fix $A \in \mathcal{P}_{d}$ and let $\delta(A)$ denote the set of all $\delta_{k}$ which can be written in the form $(9)$. Finally, for $x>0$ we set

$$
\delta(A)(x)=\left\{\delta_{k}: \delta_{k} \in \delta(A), k \leq k(x)\right\},
$$

and

$$
\pi_{\delta}(x, A)=\operatorname{card}(\delta(\{0,1,2, \ldots, k(x)\}) \cap \delta(A)(x))
$$

Since

$$
\pi_{\delta}(x)=\sum_{A \in \mathcal{P}_{d}} \pi_{\delta}(x, A),
$$

hence to finish the proof of Theorem 25 it is sufficient to show that for each fixed $A \in \mathcal{P}_{d}$ there exists a polynomial $W_{A}(x)$ such that $\operatorname{deg} W_{A}(x)<d$ and $\pi_{\delta}(x, A) \leq W_{A}(x)$. Let us fix a set $A$, let us fix a real $x>0$, and let a natural number $k$ be such that $\delta_{k} \in \delta(A)(x)$. Then, as we have observed in $(9)$,

$$
\delta_{k}=\sum_{j \in A} n_{k}^{j} \cdot a_{j}-\sum_{j \in A^{\prime}} n_{k}^{j} \cdot a_{j}=u_{k}-v_{k},
$$

where

$$
u_{k}=\sum_{j \in A} n_{k}^{j} \cdot a_{j}
$$


and

$$
v_{k}=\sum_{j \in A^{\prime}} n_{k}^{j} \cdot a_{j}
$$

Hence we have

$$
\begin{aligned}
x_{k+1} & =\sum_{j \in I_{d}} m_{k+1}^{j} \cdot a_{j} \geq \sum_{j \in A} m_{k+1}^{j} \cdot a_{j} \geq \sum_{j \in A}\left(m_{k+1}^{j}-m_{k}^{j}\right) \cdot a_{j} \\
& =\sum_{j \in A} n_{k}^{j} \cdot a_{j}=u_{k} .
\end{aligned}
$$

Thus if $x_{k+1} \leq x$, then $u_{k} \leq x$. Using the same argument (replacing $A$ by $A^{\prime}$ ) we check that $v_{k} \leq x$.

Now let $L_{A}$ denote the liken generated by those $a_{j}$ for which $j \in A$ and let $L_{A^{\prime}}$ denote the liken generated by remaining generators. Let $L^{*}=L_{A} \cup L_{A^{\prime}}$. The set $L^{*}$ is a subset of the liken $L$, then this set can be ordered as an increasing sequence. Let $L^{*}=\left(\gamma_{p}\right)_{0}^{\infty}$, and $\gamma_{0}<\gamma_{1}<\gamma_{2} \ldots$..

It follows from the above remark that for each $\delta_{k} \in \delta(A)(x)$ there exist two indices $p \in \mathbb{N}, q \in \mathbb{N}$ such that $p>q$ and by $10, \delta_{k}=\gamma_{p}-\gamma_{q}$. We will prove that $p=q+1$. Indeed, suppose that $p>q+1$. Hence we have

$$
u_{k}=\gamma_{p}>\gamma_{p-1}>\gamma_{q}=v_{k}
$$

Let $c_{k}$ denote the greatest summand of $x_{k}$ and $x_{k+1}$. Adding $c_{k}$ to both sides of the above inequality we obtain

$$
x_{k+1}=u_{k}+c_{k}>\gamma_{p-1}+c_{k}>v_{k}+c_{k}=x_{k} .
$$

Setting $y=\gamma_{p-1}+c_{k}$ we have $x_{k}<y<x_{k+1}$ which is impossible since $x_{k}$ and $x_{k+1}$ are successive in the liken $L$. Hence we proved that for each $\delta_{k} \in \delta(A)(x)$ and for each $u \in L_{A}$ and for each $v^{\prime} \in L_{A^{\prime}} \ni v^{\prime \prime}$ such $u \leq x, v^{\prime} \leq x, v^{\prime \prime} \leq x$ we have that, if $\delta_{k}=u-v^{\prime}=u-v^{\prime \prime}$ then $v^{\prime}=v^{\prime \prime}$. Thus the set $\delta(A)(x)$ has no more elements that the set

$$
U_{A}(x)=\left\{z \in L_{A}: z \leq x\right\} .
$$

Since the liken $L_{A}$ is finitely generated, the number of its elements increases like a polynomial of degree equal to the number of elements of the set $A$. But the cardinality of $A$ is less than $d$, hence we can take $W_{A}(x)=W_{\mathbb{L}_{\mathbb{A}}}$ because $\operatorname{deg} W=$ card $(A)$. This ends the proof.

\section{REMARK 26}

Theorem 25 allows us to point out once more the difference between finitely and infinitely generated likens. In the classical liken $N^{*}$ the sequence of gaps is injective, which means that $\omega_{L}(x)=k(x)=\pi_{\mathbb{L}}(x)$ (the number of different elements in the sequence of gaps grows at the same rate as the number of the elements of a liken). In consequence, $N^{*}$ cannot be finitely generated. Let us also remark that in $N^{*}$ two successive elements are always relatively prime. As we have observed in Propositions 7 and 8 , each element of a liken with uniqueness can be 
identified with the sequence of its coordinates in the expansion (2), then each element $x \in \mathbb{L}$ is a function on $\mathbb{N}$ with the values in $\mathbb{N}$. Hence it clear what we mean by the support of $x$. Thus "relatively prime" means clearly that for each $k, \operatorname{supp}\left(x_{k}\right) \cap \operatorname{supp}\left(x_{k+1}\right)=\emptyset$. This property of $N^{*}$ is never true for finitely generated likens, since in finite dimensional case there exist elements with full support.

\subsection{Some consequences of a certain theorem of Dirichlet}

We will prove some further theorems on gaps in likens for which we will need some consequences of the well known theorem of Dirichlet, see e.g. [4].

THEOREM 27

For each irrational number $\alpha$ there exists infinitely many rational numbers $\frac{p}{q}$, $p \in \mathbb{Z}, q \in \mathbb{N}$ such that

$$
\left|\alpha-\frac{p}{q}\right|<\frac{1}{q^{2}}
$$

As an easy consequence of Dirichlet's theorem we obtain

LEMMA 28

Let $\left\{a_{1}, a_{2}, \ldots, a_{k}\right\}$ be a finite sequence of positive real numbers which satisfies the following condition: for each partition of the set $\{1,2, \ldots, k\}$ onto non-empty subsets $K$ and $M$ we have

$$
\frac{\sum_{i \in K} a_{i}}{\sum_{j \in M} a_{j}} \notin \mathbb{Q}
$$

Then, for each $\eta>0$ and for each partition $(K, M)$ as above there exist infinitely many pairs $(p, q)$ of natural numbers such that

$$
\left|q \cdot \sum_{i \in K} a_{i}-p \cdot \sum_{j \in M} a_{j}\right|<\eta .
$$

Proof. Let us fix a partition $(K, M)$ and a positive number $\eta>0$. Next we apply Theorem 27 for

$$
\alpha=\frac{\sum_{i \in K} a_{i}}{\sum_{j \in M} a_{j}}
$$

and we obtain

$$
\left|\frac{\sum_{i \in K} a_{i}}{\sum_{j \in M} a_{j}}-\frac{p}{q}\right|<\frac{1}{q^{2}}
$$

for infinitely many rational numbers $\frac{p}{q}$. This implies that

$$
\left|q \cdot \sum_{i \in K} a_{i}-p \cdot \sum_{j \in M} a_{j}\right|<\frac{\sum_{i \in K} a_{i}}{q^{2}}
$$

for infinitely many $\frac{p}{q}$. We choose $q$ so large that

$$
\frac{\sum_{i \in K} a_{i}}{q^{2}}<\eta
$$

and this ends the proof. 


\subsection{Second theorem}

We will prove another theorem on gaps in likens, saying in particular, that the one-dimensional likens, in which the sequence of gaps is constant, constitute a kind of singularity. Namely, we have the following

THEOREM 29

Let $L=L\left(a_{1}, a_{2}, \ldots, a_{d}\right)=\left(x_{n}\right)_{0}^{\infty}$ denote a liken with d generators $(d>1)$. Let $\delta_{n}=x_{n+1}-x_{n}$ be a sequence of gaps of the liken $\mathbb{L}$. Then

$$
\lim _{n \rightarrow \infty} \delta_{n}=0 .
$$

Proof. It is not hard to observe that it suffices to verify our theorem only for $d=2$, but we must use also Lemma 28. Since $\delta_{n}>0$ then it is sufficient to show that

$$
\limsup _{n \rightarrow \infty} \delta_{n}=0 .
$$

To obtain a contradiction assume that there exist a number $\alpha>0$ and a subsequence $n_{1}<n_{2}<\ldots$ of the sequence of natural numbers such that $\delta_{n_{k}}>\alpha$. Then $\delta_{n_{k}}=x_{n_{k}+1}-x_{n_{k}}$. Let

$$
x_{n_{k}}=\sum_{j=1}^{d} m_{n_{k}}^{j} \cdot a_{j} .
$$

Since the sequence $x_{n_{k}}$ as a subsequence of the liken $L$ tends to infinity, at least one of $d$ sequences $m_{n_{k}}^{j}$ is a sequence of real numbers tending to infinity. We will consider two cases.

Case 1. Assume that for each $1 \leq j \leq d$ we have $\lim _{k \rightarrow \infty} m_{n_{k}}^{j}=\infty$. Let us divide the set $\{1,2, \ldots, d\}$ into two non-empty and disjoint subsets $K$ and $M$ and fix a number $\eta<\alpha$. Now we apply for Lemma $28 K$ and $L$. Consider a pair $(p, q)$ such that

$$
\left|q \cdot \sum_{i \in K} a_{i}-p \cdot \sum_{j \in M} a_{j}\right|<\eta .
$$

Without loss of generality we may assume that

$$
0<q \cdot \sum_{i \in K} a_{i}-p \cdot \sum_{j \in M} a_{j}<\eta
$$

Now we choose $n_{k}$ so large that $1 \leq j \leq d$ there is $m_{n_{k}}^{j}>\max (p, q)$. Consider a point

$$
x=\sum_{i \in K}\left(m_{n_{k}}+q\right) \cdot a_{i}+\sum_{j \in M}\left(m_{n_{k}}-p\right) \cdot a_{j} .
$$

Since all coefficients at the generators are positive then $x$ belongs to the liken $L$ and there exists $s \in \mathbb{N}$ such that $x=x_{s}$. We will prove that $x_{n_{k}}<x_{s}$. Indeed, we have

$$
x_{s}-x_{n_{k}}=q \cdot \sum_{i \in K} a_{i}-p \cdot \sum_{j \in M} a_{j}>0 .
$$


In consequence, we have

$$
\alpha<\delta_{n_{k}}=x_{n_{k}+1}-x_{n_{k}} \leq x_{s}-x_{n_{k}}=q \cdot \sum_{i \in K} a_{i}-p \cdot \sum_{j \in M} a_{j}<\eta \leq \alpha .
$$

This contradiction ends the proof of the Case 1.

Case 2. Let us suppose that there exists $j$ such that the sequence $m_{n_{k}}^{j}$ is bounded and let $K$ be the set of all such $j$. More exactly, $K:=\left\{j: m_{n_{k}}^{j}<A_{j}\right\}$. It follows from our assumption that $M=\{1,2, \ldots, d\} \backslash K \neq \emptyset$. We choose $\eta<\alpha$ and apply Lemma 28 for $K, M$ and $\eta$ as above. We choose a pair $(p, q)$ such that we have the inequality

$$
0<q \cdot \sum_{i \in K} a_{i}-p \cdot \sum_{j \in M} a_{j}<\eta
$$

and we choose $n_{k}$ such that $m_{n_{k}}^{j}>p$ for $j \in M$.

Now we set

$$
x=\sum_{i \in K}\left(m_{n_{k}}+q\right) \cdot a_{i}+\sum_{j \in M}\left(m_{n_{k}}-p\right) \cdot a_{j}
$$

and we use the same argument as in Case 1. Since all coefficients at generators are positive then $x$ belongs to $L$ and $x=x_{s}$ for some $s \in \mathbb{N}$. We check that $x_{n_{k}}<x_{s}$. Indeed, we have

$$
x_{s}-x_{n_{k}}=q \cdot \sum_{i \in K} a_{i}-p \cdot \sum_{j \in M} a_{j}>0 .
$$

In consequence,

$$
\alpha<\delta_{n_{k}}=x_{n_{k}+1}-x_{n_{k}} \leq x_{s}-x_{n_{k}}=q \cdot \sum_{i \in K} a_{i}-p \cdot \sum_{j \in M} a_{j}<\eta \leq \alpha
$$

and this contradiction ends the proof.

\section{REMARK 30}

Theorem 29 may by interpreted geometrically. As we have proved in Theorem 22 the function $\pi_{\mathbb{L}}(x)$ behaves like a polynomial $W_{\mathbb{L}}$ which has the degree equal to the dimension of $\mathbb{L}$. Hence the number of the elements of $\mathbb{L}$ in an interval $[x, x+1]$ behaves like the derivative $W_{\mathbb{L}}^{\prime}$ of $W_{\mathbb{L}}$, hence tends to infinity with $x$ and, in consequence, the average gap tends to 0 . Theorem 29 says that, in some sense, there are no irregularities in the sequence of gaps.

\section{References}

[1] Beurling, Arne. "Analyse de la loi asymptotique de la distribution des nombres premiers généralisés. I." Acta Math. 68, no. 1 (1937): 255-291. Cited on 96. 
[2] Diamond, Harold G., and Wen-Bin Zhang. Beurling generalized numbers. Vol 213 of Mathematical Surveys and Monographs. Providence, RI: American Mathematical Society, 2016. Cited on 96 .

[3] Ehrhart, Eugène. "Sur les polyèdres rationnels homothétiques à n dimensions." $C$. R. Acad. Sci. Paris 254 (1962): 616-618. Cited on 108.

[4] Rose, Harvey E. A course in number theory. Second edition. New York: The Clarendon Press; Oxford University Press, 1994. Chapter 13. Cited on 112.

\author{
State Higher Vocational School in Tarnów \\ ul. Adama Mickiewicza 8 \\ 33-100 Tarnów \\ Poland \\ E-mail: Edward.Tutaj@im.uj.edu.pl
}

Received: November 5, 2017; final version: November 29, 2017; available online: January 15, 2018. 